\title{
Hundimiento de las aguas al sur de Cuba
}

\author{
Benigno Hernández ${ }^{1,2}$ \& Roberto Piñeiro ${ }^{1}$ \\ ${ }^{1}$ Centro de Investigaciones Pesqueras (CIP), Ciudad de la Habana, Cuba \\ ${ }^{2}$ Dirección actual: CICESE, División de Oceanología, P.O. Box 434844, San Diego \\ CA. 92143-4844, USA \\ E-mail: bhernan@cicese.mx
}

\begin{abstract}
RESUMEN. Se realizan 23 estaciones oceanográficas a bordo del Buque de Investigaciones Científicas BIC "Ulises" en marzo de 1994 y abril de 1996 en el polígono de estudio situado en los $21^{\circ} 00^{\prime}-21^{\circ} 30^{\prime} \mathrm{N}$ y $81^{\circ} 50^{\prime}-83^{\circ} 00^{\prime} \mathrm{W}$ al sureste de la Isla de la Juventud, Cuba, con el objetivo de estudiar algunas de las relaciones hidrofísicas que controlan la dinámica de las larvas de langosta espinosa Panulirus argus en la interfase del talud con el océano. Se analizan el viento, transporte Ekman, turbulencia, profundidad de inicio de la termoclina, temperatura superficial del mar y estabilidad vertical en el hundimiento de las aguas cercanas al talud en los dos cruceros de primavera. El índice de transporte (IT) mostró un movimiento neto hacia la costa, provocando un hundimiento en la zona cercana al talud. El hundimiento produce un efecto de mezcla o falso afloramiento llamado "efecto cascada" que mantiene los nutrientes resuspendidos por un período que varía según la intensidad del viento y las corrientes imperantes en la zona. Este efecto, al parecer, es responsable de la retención larvaria en esta región
\end{abstract}

Palabras claves: hundimiento, transporte Ekman, estabilidad vertical, estrés del viento, termoclina, temperatura superficial del mar, langosta.

\section{Downwelling waters in the south of Cuba}

\begin{abstract}
Take account 23 oceanographic stations on Oceanographic Research Vessel BIC "Ulises" in March 1994 and April 1996 in the study polygon located in the $21^{\circ} 00^{\prime}-21^{\circ} 30^{\prime} \mathrm{N}$ and $81^{\circ} 50^{\prime}-83^{\circ} 00^{\prime} \mathrm{W}$ in southwest of Isla de la Juventud, Cuba, with the main objective to study hydrophysical relationships that control the dynamics of the Panulirus argus spiny lobster larvae in the boundary shelf. Importance of the wind, Ekman transport, turbulence, depth of the top of the thermocline, sea surface temperature, vertical stability in the convergence or downwelling nearshore waters in this important area of spawns, was demonstrated. Transport Index (IT) showed a net movement toward the coast causing nearshore downwelling. The downwelling produces a mixture effect or false upwelling called also "cascade effect" that maintains nutrients suspended for one period that varies according to the wind intensity and the prevailing currents in the area. This effect apparently, is responsible for the larvae retention in this region.
\end{abstract}

Key words: downwelling, Ekman transport, vertical stability, wind stress, thermocline, sea surface temperature, spiny lobster.

\section{INTRODUCCIÓN}

Mientras que en el mundo se estudian y reportan los aspectos físicos y biológicos más relevantes asociados a pelágicos menores relacionados con grandes zonas de divergencia o afloramientos (Kucas, 1986; Mysak, 1986; Bakun et al., 1992; Francis \& Hare, 1994), no es común encontrar trabajos que relacionen estas investigaciones en áreas donde existe lo contrario, como son las zonas de convergencia o hundimiento, situación que no favorece al enrique- cimiento trófico sostenido de las poblaciones planctónicas.

La convergencia de las aguas del mar o hundimiento costero provocado por la deriva superficial o transporte Ekman (1905), es un proceso fluctuante que tiene su origen en la fuerza y dirección del viento y puede tener efectos relevantes en los recursos pesqueros de cualquier región (Fofonoff \& Montgomery, 1955). 
Las primeras informaciones sobre afloramientos y hundimientos a partir del cálculo de las corrientes geostróficas alrededor de Cuba comenzaron en los años 60-70, cuando diversos autores (Elizarov \& Gómez, 1966; Bogdanov, 1966; Rossov, 1967; Emilsson \& Gómez, 1971; Gómez, 1979), entregaron sus primeras impresiones sobre centros ciclónicos y anticiclónicos en aguas cercanas a Cuba. Posteriormente, Siam \& Hernández (1981) y Rodríguez et al. (1990), ayudaron a esclarecer y localizar también a partir de corrientes geostróficas, algunos de estos tipos de oscilación en la vertical mediante el panorama circulatorio en la zona económica exclusiva (ZEE) de Cuba. Sin embargo, las investigaciones sobre los afloramientos costeros en aguas cubanas, relacionados con la fuerza ondulatoria del viento que sopla sobre la superficie del mar cercano al talud, y que tienen su origen en la deriva superficial de Ekman, sólo fueron tratadas años atrás por Tápanes (1963).

El papel que desempeña el transporte Ekman y la turbulencia en el hundimiento de las aguas al sur del golfo de Batabanó, relacionados con la estancia larvaria de los primeros estadios de filosomas de langosta Panulirus argus debido a la profundización de la termoclina (PIT) y a la isoterma de $26^{\circ} \mathrm{C}$, ha sido analizada en los trabajos de Hernández et al. (1995) y Alfonso et al. (1995a).
El propósito del presente trabajo es construir una base para la aplicación de métodos comparativos entre los mecanismos océano-plataformas restantes de la isla de Cuba, para identificar las relaciones hidrofísicas que controlan la dinámica de la fauna en la interfase del talud con la plataforma marina, entre ellas, las larvas de langosta $P$. argus. En este sentido, se analiza el ambiente oceanográfico y las condiciones hidroclimáticas derivadas del hundimiento de las aguas al sureste de Punta del Este, región suroccidental de Cuba, que corresponde a una de las principales áreas de desove de la langosta.

\section{MATERIALES Y MÉTODOS}

El estudio se realizó en el área conformada por el polígono situado entre los $21^{\circ} 00^{\prime}-21^{\circ} 30^{\prime} \mathrm{N}$ y $81^{\circ} 50^{\prime}$ $83^{\circ} 00^{\prime} \mathrm{W}$ al sureste de la Isla de la Juventud región suroccidental de Cuba, a bordo del BIC "Ulises" en marzo de 1994 y abril de 1996. La primera estación se situó a dos millas de la costa, aproximadamente en los $82^{\circ} 35^{\prime} \mathrm{W}$ frente al Faro Carapachibey, al sur de la Isla de la Juventud, mientras que el último transecto se situó igualmente a dos millas al sur de Cayo Largo del Sur, en el Archipiélago de los Canarreos (Fig. 1).

En el crucero de marzo de 1994 se realizaron cuatro transectos, desde el segundo al quinto, con

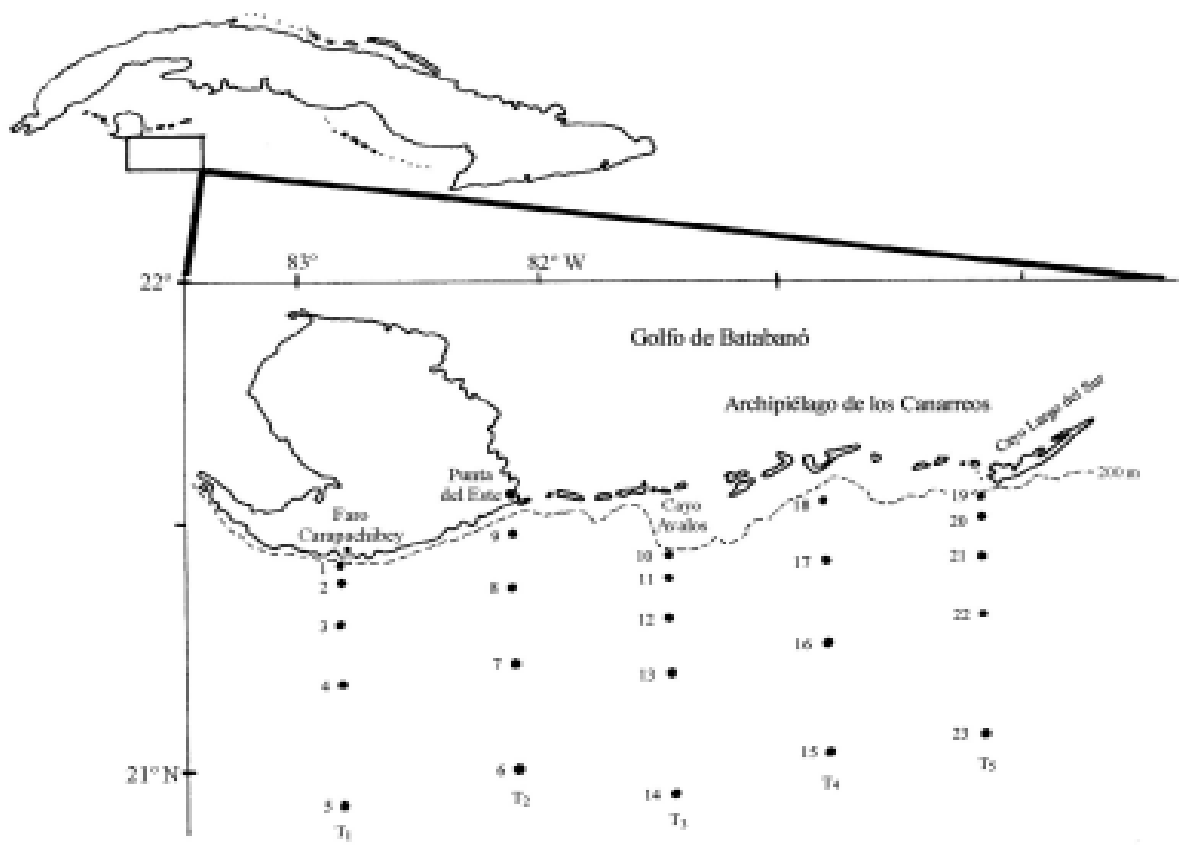

Figura 1. Red de estaciones y transectos en el polígono de estudio al sureste del golfo de Batabanó, región suroccidental de Cuba, durante los cruceros efectuados en marzo de 1994 y abril de 1996.

Figure 1. Transects and stations grid in the study polygon in the southeast of the Gulf of B a $\mathbf{t}$ a b a n o, southwest e r n region of Cuba, during March 1994 and April 1996 cruises. 
cinco estaciones en los transectos impares (III y V) y cuatro estaciones en los transectos pares (II y IV), estas últimas de más de 24 horas de duración o estaciones fijas. En el crucero de 1996 se realizaron cinco transectos con las estaciones señaladas anteriormente. En total se realizaron 50 estaciones oceanográficas en el crucero de 1994 y 56 en 1996. En las estaciones fijas, los muestreos se realizaron a las 09, 15, 21, 03 y $09 \mathrm{~h}$ (referidas al uso horario del meridiano $75^{\circ} \mathrm{W}$ ), ésta última fue para cerrar el ciclo de inicio en cada horario de arribo.

En cada una de las estaciones se realizaron lances de botellas Nansen de $1 \mathrm{~L}$, con termómetros de inversión protegidos, con precisión de $0,02^{\circ} \mathrm{C}$, a 12 niveles de profundidad: $0,15,25,50,75,100,125$, $150,175,200,250$ y $300 \mathrm{~m}$. La salinidad se determinó con un salinómetro inductivo del orden de las centésimas y se calculó la densidad para verificar la profundidad de inicio de la termoclina (PIT) en todos los transectos. Los datos se procesaron mediante el programa DATAOCEAN (IDO, 1989), que calcula entre otros, el gradiente mínimo para determinar la PIT.

Se consideró la rosa de los vientos en la estación meteorológica de Punta del Este, región suroccidental de la Isla de la Juventud (Vega, 1989), mostrando que la mayor parte del territorio sur de la isla de Cuba, presenta el régimen de vientos alisios ó predominantes de componente este, interrumpido por fluctuaciones atmosféricas que acontecen a lo largo del año alterando la marcha normal de la componente señalada. Estas fluctuaciones se deben al paso de frentes fríos que hacen girar los vientos del $1^{\circ}$ al $4^{\circ}$ cuadrante; bajas invernales que se mueven sobre el golfo de México y que hacen girar los vientos al sur; tormentas locales severas y tornados; hondonadas y ondas tropicales; paso de tormentas tropicales y huracanes (Hernández, 2002).

Para el cálculo del Índice de Transporte (IT) y de turbulencia se utilizó la metodología empleada por Bakun (1973) para la costa oeste de América del Norte basado en la teoría de Ekman (1905), bajo la asunción de un movimiento uniforme estable y un océano infinito. Este índice es una expresión cuantitativa de transporte de masa de agua con referencia a la línea de costa. Por consiguiente, el transporte (E) se calcula usando la fuerza del viento (t) sobre la superficie y los parámetros de Coriolis (f) del área:

$$
\mathrm{E}=\mathrm{t} / \mathrm{f}
$$

La fuerza del viento (wind stress) está dada por la expresión:

$$
\mathrm{t}=\mathrm{Ba} \cdot \mathrm{CD} \cdot \mathrm{V}^{2}
$$

donde

ßa : es la densidad del aire $\left(1,2 \mathrm{~kg} \cdot \mathrm{m}^{-3}\right)$

$\mathrm{CD}$ : coeficiente empírico de arrastre del viento para situaciones estables $(0,0013)$ basado en la teoría de Charnock (1955, 1981) y Banner (1990).

$\mathrm{V}$ : velocidad del viento

El patrón de Coriolis depende de la latitud del área y se calcula según la expresión:

$$
\mathrm{f}=2 \mathrm{~W} \cdot \operatorname{sen} \theta
$$

donde

W : velocidad angular de la rotación de la tierra $\left(7,2921 \cdot 10^{-5} \mathrm{rad} \cdot \mathrm{s}^{-1}\right)$

$\theta$ : latitud en grados

El Índice de Transporte (IT) equivale a:

$$
\mathrm{IT}=\mathrm{E} \cdot \cos \delta
$$

donde $\delta$ es el ángulo de la costa con respecto a la dirección donde sopla el viento, quedando la fórmula general como, uniendo (1) con (2):

$$
\mathrm{E}=\cos \delta\left[ß \mathrm{a} \cdot \mathrm{CD} \cdot \mathrm{V}^{2}\right] / 2 \mathrm{~W} \cdot \operatorname{sen} \theta
$$

Las unidades del IT son $\mathrm{t} \cdot \mathrm{s}^{-1} \cdot 100 \mathrm{~m}$ de costa. La turbulencia en la columna superior de agua está dada por la expresión $\mathrm{V}^{3}$. Esta información se archivó en soporte magnético del programa Bakun creado para tal efecto (Pérez \& Alfonso, 1991).

Los datos meteorológicos a bordo del BIC "Ulises" se registraron cada hora y durante todo el tiempo que duró el crucero, éstos incluyen la dirección y velocidad del viento, cielo cubierto, tipo de nubes, temperatura húmeda y seca, humedad relativa, presión atmosférica, así como otros eventos de tipo sinóptico que afectaron la zona de estudio. Con estos resultados se prepararon cartas horizontales de cobertura nubosa, fuerza de ondulación del viento, índices de turbulencia y transporte, temperatura superficial del mar (TSM), salinidad y PIT. Además, en ambos cruceros se prepararon perfiles verticales de temperatura del mar en los transectos II y IV.

Se realizó el cálculo de la estabilidad vertical desde 0 a $300 \mathrm{~m}$ de profundidad en intervalos pre- 
viamente establecidos: 0-10, 10-25, 25-50, 50-75, 75-100, 100-150, 150-200 y 200-300 m, según la siguiente expresión (Egorov, 1983):

$$
\psi=[\Delta \mathrm{p} / \Delta \mathrm{z}] / 10^{-5}
$$

donde

$\psi$ : estabilidad

$\Delta \mathrm{p}$ : gradiente de densidad en los intervalos de profundidad seleccionados

$\Delta \mathrm{z}$ : gradiente de profundidad de cada intervalo

Se confeccionaron perfiles verticales de estabilidad y de la PIT en las estaciones 9 y 18, que fueron las más costeras de los transectos fijos II y IV, respectivamente.

\section{RESULTADOS}

\section{Cobertura nubosa}

En marzo de 1994 en horas de la mañana predominó el equilibrio entre días nublados y despejados (Fig. 2). En la tarde predominaron los días nublados con $66 \%$ del total de la información obtenida y el resto estuvo despejado. Durante la noche y en la madrugada prevaleció el cielo despejado con $63 \mathrm{y}$ $57 \%$ respectivamente. En el transecto II, estación 12, fueron los más despejados y en el resto de las estaciones estuvo nublado en algún momento con un incremento casi total de nubes en la estación 21.

En abril de 1996 en horas de la tarde igualmente predominó el equilibrio entre días nublados y despejados (Fig. 2). Durante la tarde, noche y madrugada prevalecieron los días despejados con 54, 67 y $72 \%$ respectivamente, siendo la 1 y la 14 las estaciones con cielo más despejado, mientras que los nublados correspondieron a la estación 3.

En abril de 1996 la cobertura nubosa disminuyó con respecto a marzo de 1994 , lo que permitió mayor penetración de la radiación solar en el mar, con el consiguiente aumento de la temperatura del día y disminución de la misma en la noche. En este senti- do, la nubosidad no deja escapar las ondas largas de temperatura que despide el océano y que se incrementan en la noche, atrapándolas y manteniendo alta la temperatura en superficie. Por lo tanto, existió una relación casi directa entre el aumento de los días nublados y el aumento de la TSM, correspondiendo los núcleos de mayor temperatura con un máximo de nubosidad y viceversa.

Desde el punto de vista sinóptico, la cobertura nubosa en la región de Punta del Este se caracterizó por un patrón estacional bien definido de días nublados y despejados. Según Seguí (1989), el mayor porcentaje de días nublados ocurrió en septiembre (55\%), mientras que en abril se presentó el mayor porcentaje de días despejados (37\%) (Tabla 1), siendo también en este mes donde se produce la mayor radiación solar directa y difusa (Campos, 1989), con $11 \mathrm{~h} \mathrm{luz} \cdot \mathrm{día}^{-1}$.

En primavera, el patrón de isopletas de radiación solar sobre el mar, disponible para la absorción, ya sea por el agua o por el fitoplancton, está fuertemente favorecido por la escasa distribución de la cobertura nubosa en marzo-abril.

\section{Fuerza del viento}

En marzo de 1994 el viento fue del primer cuadrante o del noreste en el transecto $\mathrm{V}$, mientras que en los otros transectos del polígono de estudio, el viento sopló del segundo cuadrante. Las mayores intensidades se registraron en la estación 18, mientras que las menores en las estaciones 13 y 14 (Fig. 3).

Sin embargo, en abril de 1996 fue más intenso que en el crucero anterior, con 0,009 unidades de fuerza del viento (UFV) como promedio, contra 0,007 en 1994. Es decir, 0,002 UFV menos que en 1996 (Fig. 3). En el primer y último transecto, el viento sopló del segundo cuadrante, mientras que en el resto lo hizo del primero, preferentemente de $\operatorname{los} 90^{\circ}$. Los vientos fueron más fuertes en las estaciones 10 y 11 , a partir de aquí se genera una intensa mezcla en el agua con el consiguiente aumento de la turbulencia, transporte y disminución de la PIT.

Tabla 1. Porcentaje de días nublados y despejados en la zona de Punta del Este.

Table 1. Percentage of cloudy days and cloudless in Punta del Este area.

\begin{tabular}{|lcccccccccccc|}
\hline Mes & Ene & Feb & Mar & Abr & May & Jun & Jul & Ago & Sep & Oct & Nov & Dic \\
\hline Nublados & 24 & 20 & 23 & 20 & 34 & 50 & 52 & 52 & 55 & 45 & 35 & 20 \\
Despejados & 30 & 33 & 33 & 37 & 20 & 12 & 15 & 15 & 12 & 18 & 25 & 30 \\
\hline
\end{tabular}



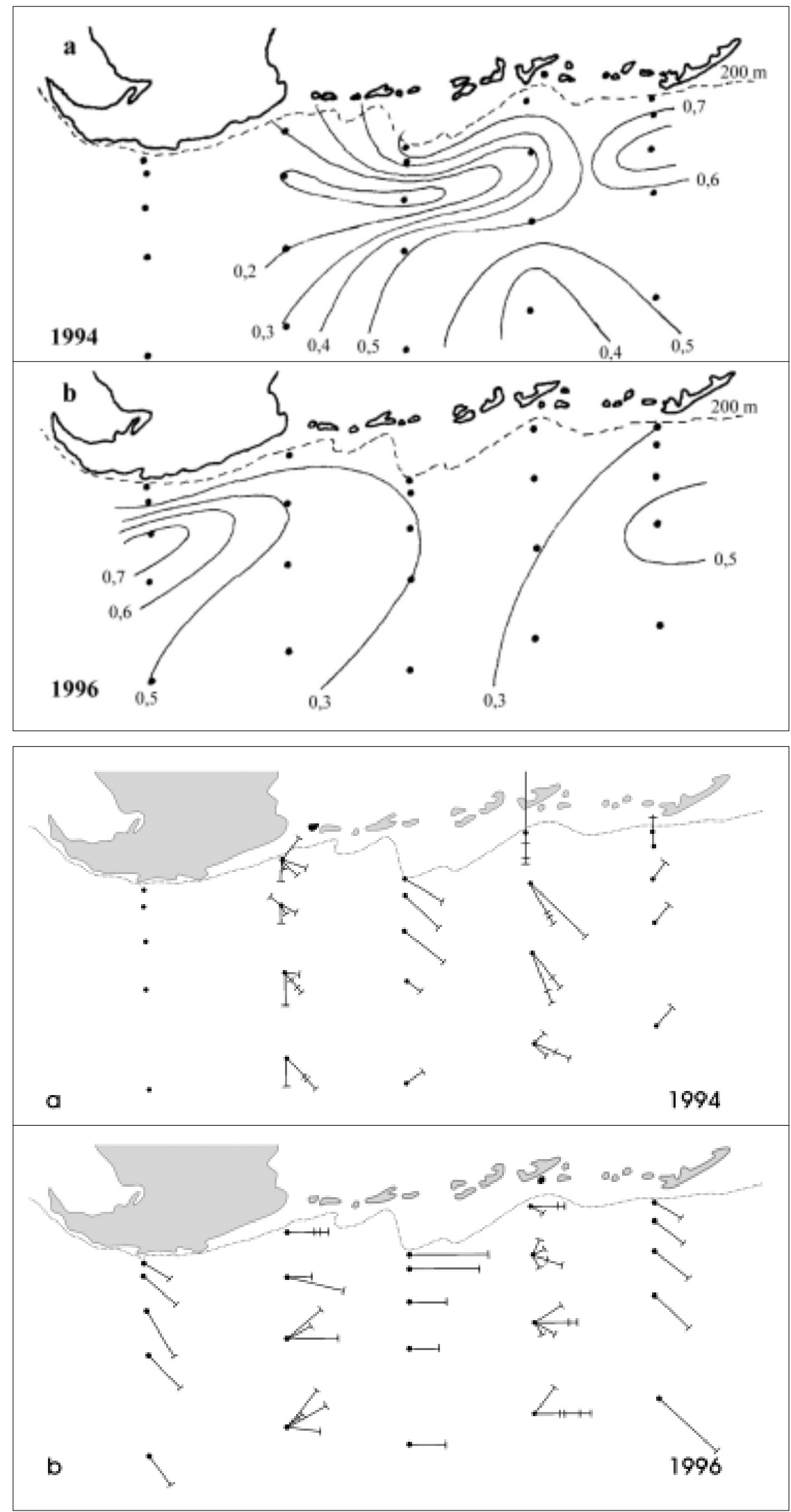

Figura 2. Distribución de la cobertura nubosa (CN). a) Crucero de marzo 1994, b) Crucero de abril 1996. Las unidades de la distribución de la CN están expresadas en octavos divididos entre ellos mismos.

Figure 2. Cloudy covering $(\mathrm{CN})$ distribution. a) March 1994 cruise, b) April 1996 cruise. Cloudy covering unit's distributions are expressed in eighth divided among them.

Figura 3. Distribución superficial de la fuerza del viento. a) Crucero de marzo 1994, b) Crucero de abril 1996. Las unidades de la fuerza del viento (UFV) están expresadas en dinas por $\mathrm{cm}^{2}$.

Figure 3. Wind stress curl surface distribution. a) March 1994 cruise, b) April 1996 cruise. Wind force units (UFV) are expressed in dines $\mathrm{cm}^{-2}$. 
La velocidad media del viento en ambos cruceros fue de $5 \mathrm{~m} \cdot \mathrm{s}^{-1}$ (Fig. 7). La velocidad media del viento indica que en el período de noviembre-abril, se registraron los máximos valores, especialmente en marzo-abril (Rodríguez \& Ballester, 1985); mientras que los mínimos se registraron en septiembre.

\section{Índice de Transporte (IT)}

Los resultados del Índice de Transporte reflejan, entre otros elementos, la movilidad del campo hidrológico, el movimiento del régimen superficial y subsuperficial de la temperatura del agua, la estratificación y las características del medio físico afectando o favoreciendo la supervivencia de organismos marinos en varias fases de su ciclo de vida.

El IT mostró un movimiento neto hacia la costa, provocando un intenso y perenne hundimiento en zonas cercanas al talud, reflejado en todas las variables analizadas en ambos cruceros (Figs. 4a y 4b).

Es válido destacar que la dirección del IT en ambos cruceros no es la misma. De forma general, se apreció un comportamiento este-noreste en 1994 (Fig. 4a), recayendo los mayores valores en las estaciones $10,17.3$ y 18.5 (Fig. 7a); mientras que en
1996 la tendencia general fue hacia el norte, perpendicular al talud, reforzando aún más el hundimiento costero (Fig. 4b). En 1996 los mayores valores se reportaron en las estaciones 10 y 11 , con un máximo en la estación 10 (Fig. 7b). En las estaciones de más de 24 h se observó una similitud entre ellas al no diferir mucho la intensidad del transporte. El valor promedio del IT en todas las estaciones realizadas fue de $7,0 \cdot 10^{2}$ en marzo de 1994 y $8,5 \cdot 10^{2}$ en abril de 1996 (Figs. 7a y 7b).

El hecho que se intensifique el hundimiento por ritmos irregulares del viento de varios días de persistencia, ocasionado por desórdenes atmosféricos cercanos, no provoca la desertificación de parches alimentarios en una zona a escasas millas del talud. Por el contrario, intensifica la salida de agua por el fondo del golfo de Batabanó, integrándose a una importante zona de mezcla, simulando un afloramiento muy próximo a la nutriclina.

La zona de mezcla cargada de nutrientes y elevado potencial alimenticio para las larvas será más accesible a estas últimas, cuando haya finalizado el período tormentoso o el viento soplando 4-5 días menor de $5 \mathrm{~m} \cdot \mathrm{s}^{-1}$, llamado también eventos "Lasker" (Pauly, 1989). Bakun \& Parrish (1991) reportaron

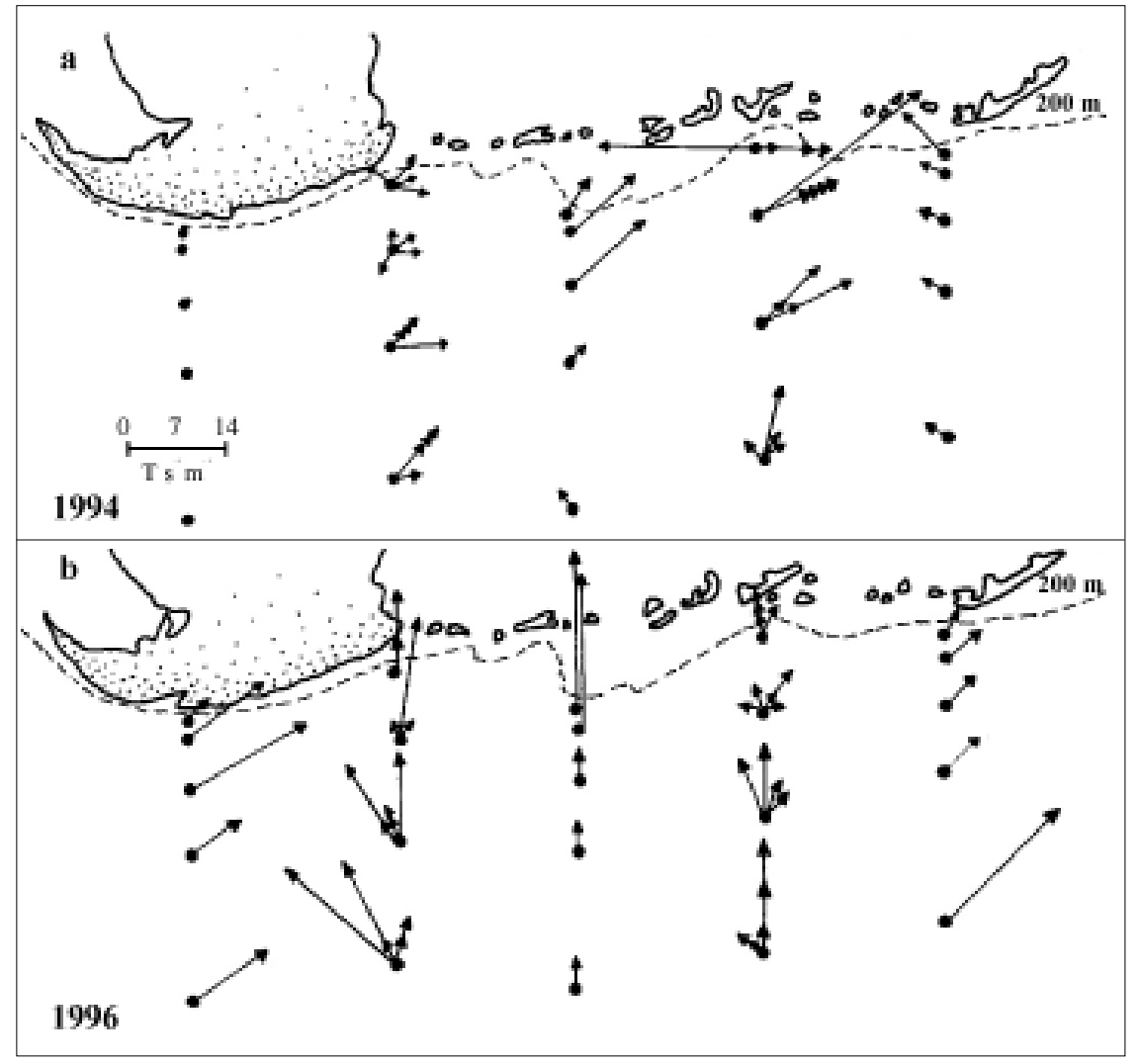

Figura 4. Distribución del Índice de Transporte Ekman. a) Crucero de marzo 1994, b) Crucero de abril 1996. Nótese que el movimiento general del transporte es hacia la costa, provocando hundimiento.

Figure 4. Transport Ekman's Index Distribution. a) March 1994 cruise, b) April 1996 cruise. See that the general transport motion is toward the coast causing downwelling. 
frente a la costa de Argentina un efecto similar al de simulación de afloramiento, para la anchoveta Engraulis anchoita.

\section{Índice de Turbulencia}

Este índice que de forma cuantitativa se mide como la velocidad del viento al cubo, mostró la existencia de mayor turbulencia, como promedio, cercano a la costa (Figs. 5a y 5b), mientras que más alejado de la misma, la turbulencia decreció. Durante 1994 las estaciones 11,12 y 17 reportaron los mayores índices de turbulencia, mientras que en 1996 recayeron en las estaciones 3, 10, 11 y 23.

Por lo tanto, en las estaciones donde se registró la mayor turbulencia, se produjo un elevado momento de mezcla que propició la dispersión de larvas y alimento en el agua. Lasker (1975) y Owen (1989) encontraron que los eventos fuertes de viento disipan las capas de alimento planctónico. En ambos cruceros, las estaciones del transecto II reportaron períodos de poco viento (menos de $5 \mathrm{~m} \cdot \mathrm{s}^{-1}$ ) y turbulencia reducida, pudiendo favorecer la concentración de larvas y permanencia de las mismas por un período adecuado que satisfaga su momento nutricional.

\section{Profundidad de Inicio de la Termoclina (PIT)}

En océanos abiertos, la tendencia general de la PIT con relación al viento, es profundizarse cuando la velocidad del viento es máxima, a disminuir poco cuando el viento es moderado y a desaparecer el inicio de la termoclina cuando existe calma (Munk \& Anderson, 1948). Sin embargo, la distribución horizontal de la PIT en marzo de 1994 y abril de 1996, muestra que cuando aumenta la velocidad del viento la termoclina cercana al talud tiende a disminuir en profundidad (Fig. 6). El hecho que la termoclina se encuentre a profundidades menores, responde a que en la zona próxima al talud, al aumentar la velocidad del viento aumenta igualmente el índice de transporte hacia la costa, eleva el nivel medio del mar y el hundimiento originado crea un efecto de mezcla de abajo hacia arriba parecido a un afloramiento, que hace disminuir en profundidad la PIT haciéndola más superficial. Por el contrario, cuando disminuye la velocidad del viento o de transporte, la PIT cercana al talud tiende a profundizarse.

En la Figura 7 se observa la serie de la velocidad del viento, transporte Ekman y PIT, para cada estación en los cruceros estudiados. Se observó que cuando aumenta la velocidad del viento por encima de $5 \mathrm{~m} \cdot \mathrm{s}^{-1}$, la termoclina disminuye en profundidad y viceversa, mientras que el índice de transporte aumenta igualmente. La PIT osciló como promedio sobre los $144 \mathrm{~m}$ y los $163 \mathrm{~m}$ en marzo de 1994 y abril de 1996, respectivamente.

\section{Temperatura Superficial del Mar (TSM)}

La distribución horizontal de la TSM presentó características homogéneas en marzo de 1994 y abril de 1996 (Fig. 8). En el transecto IV se observaron núcleos de agua más cálidas, mientras que en el transecto II fueron menos cálidas. El diapasón de temperatura superficial varió entre 26,6 y $27,6{ }^{\circ} \mathrm{C}$ para ambos cruceros, reportándose los mayores valores de temperatura frente a Cayo Rosario y los menores al sur de Punta del Este.

Esta diferencia de temperatura ó frente térmico, bien delimitada en el transecto III ó plataforma saliente al sur de Cayo Ávalos, se pudo originar por la penetración más al este de la contracorriente cubana que baja del $\mathrm{N}$ más fría, bordeando la costa sur del golfo de Batabanó causando el descenso de temperatura al sur de Isla de la Juventud. Esto debe tomarse con cautela ya que no ha sido confirmado por equipos de medición pero responde al patrón señalado por Gómez (1979).

La Figura 9 mostró la distribución vertical de las isotermas en los transectos fijos II y IV en ambos cruceros y los horarios de muestreo establecidos. Se observó el efecto de mezcla que se origina en el transecto II, cercano al talud en la madrugada (03 h) cuando regularmente el viento alcanza su máxima velocidad. Las isotermas ubicadas cerca del talud por encima de $100 \mathrm{~m}$ afloran después a escasas millas fuera del talud (Figs. 9a y 9c). En horas de la mañana (09 h), la mezcla tiende a cesar, ya que el viento disminuye notablemente hasta casi cercano a la calma. Esto ocurre con mayor intensidad en los primeros $150 \mathrm{~m}$, y aunque en 1994 estuvo más desaparecido que en 1996, aún permanece en este último año, pero con menor intensidad que en horas de la madrugada.

Durante el crucero de marzo de 1994 en horas de la tarde $(15 \mathrm{~h})$, las isotermas mantuvieron el hundimiento cercano al talud cercano a los $50 \mathrm{~m}$ en el transecto II (Fig. 9a), con poco efecto de mezcla por encima de los $100 \mathrm{~m}$, a pesar que al mediodía comenzó regularmente el "brisote", viento ligero que en ocasiones llega a ser fuerte en la interfase martierra. En el mismo transecto, pero en abril de 1996 

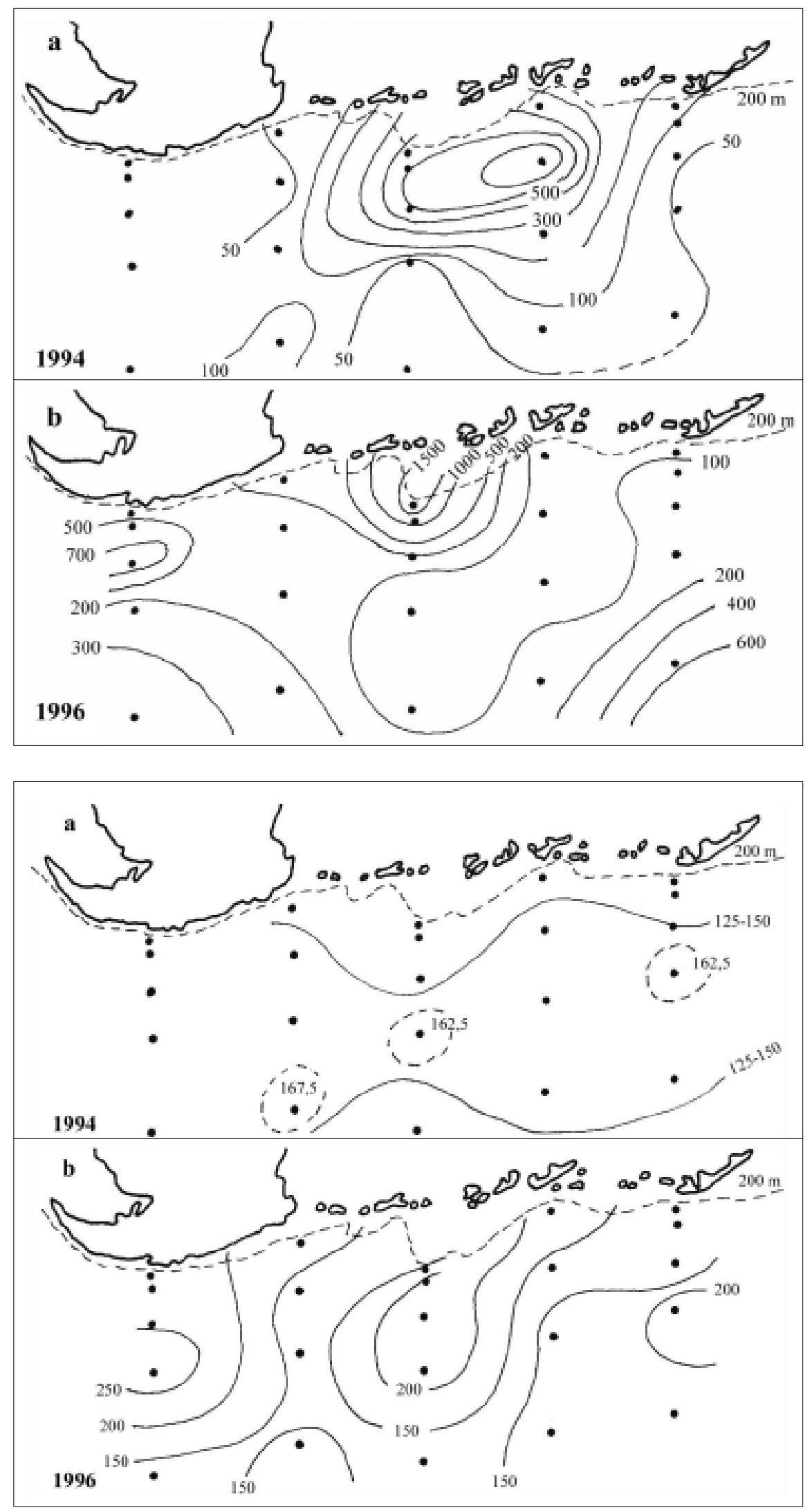

Figura 5. Distribución superficial de la turbulencia. a) Crucero de marzo 1994, b) Crucero de abril 1996. Las unidades de la turbulencia están expresadas por la velocidad del viento al cubo $\left(V^{3}\right)$. Nótese como la turbulencia es mayor como promedio en la zona costera.

Figure 5. Turbulence surface distribution. a) March 1994 cruise, b) April 1996 cruise. Turbulence units are expressed by the cube of the wind speed $\left(V^{3}\right)$. Turbulence is higher as average in the coastal area.

Figura 6. Distribución horizontal de la profundidad de inicio de la termoclina (PIT). a) Crucero de marzo 1994, b) Crucero de abril 1996. Las unidades de la distribución de la PIT están expresadas en metros.

Figure 6. Top of the thermocline horizontal distribution (PIT). a) March 1994 cruise, b) April 1996 cruise. PIT units distributions are expressed in meters. 


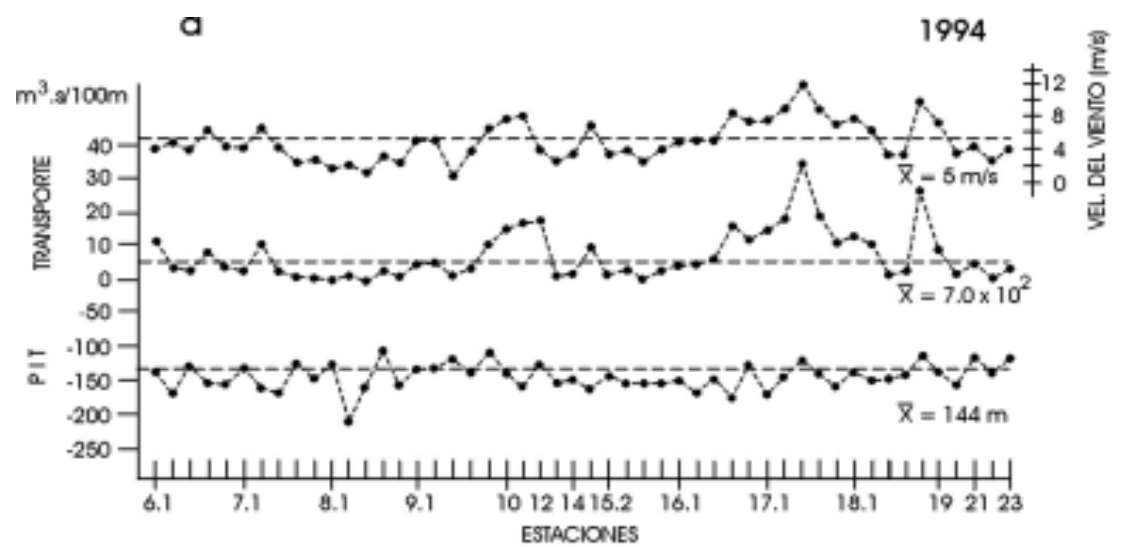

b
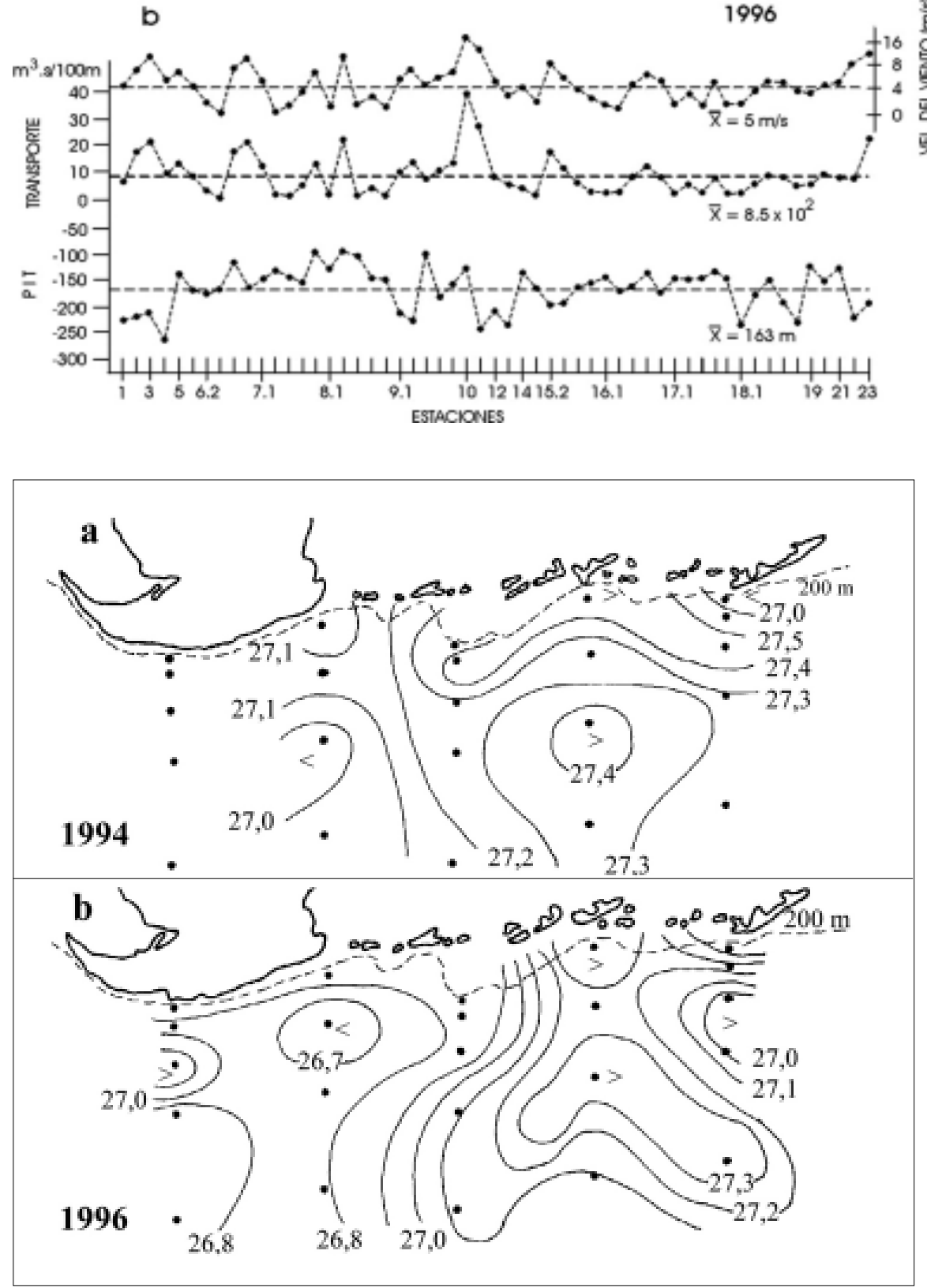

Figura 7. Serie de tiempo de velocidad del viento, índice de transporte y profundidad de inicio de la 줄 termoclina (PIT), en cada una de las estaciones en los cruceros de marzo 1994 (arriba) y abril 1996 (abajo).

Figure 7. Wind speed time series, transport index and top of the thermocline (PIT) for all stations in the cruises of March 1994 (top) and April 1996 (below).

Figura 8 Distribución horizontal de la temperatura superficial del mar (TSM). a) Crucero de marzo 1994, b) Crucero de abril 1996. Las unidades de la distribución de la TSM están expresadas en grados Celsius $\left({ }^{\circ} \mathrm{C}\right)$.

Figure 8. Sea surface temperature (SST) horizontal distribution. a) March 1994 cruise, b) April 1996 cruise. SST units are expressed in Celsius degrees $\left({ }^{\circ} \mathrm{C}\right)$. 

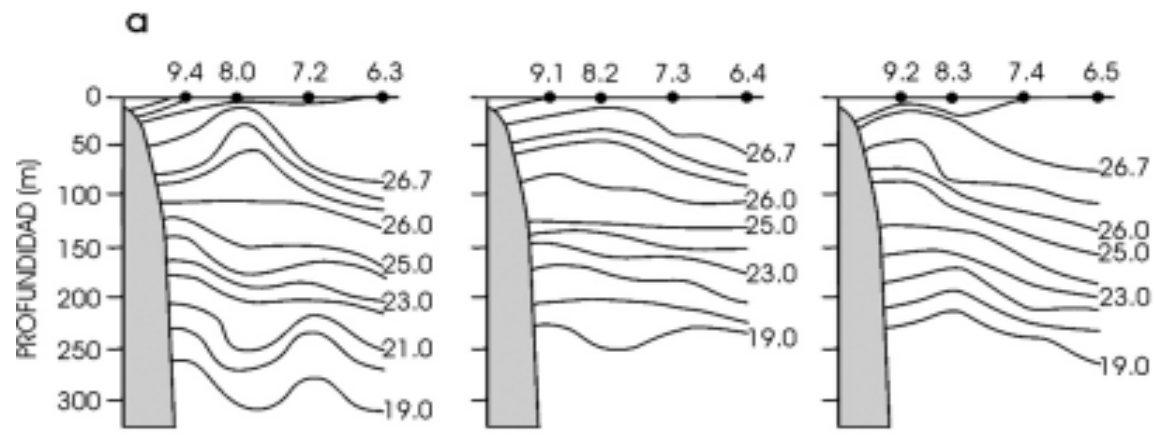

TRANSECTO II

b
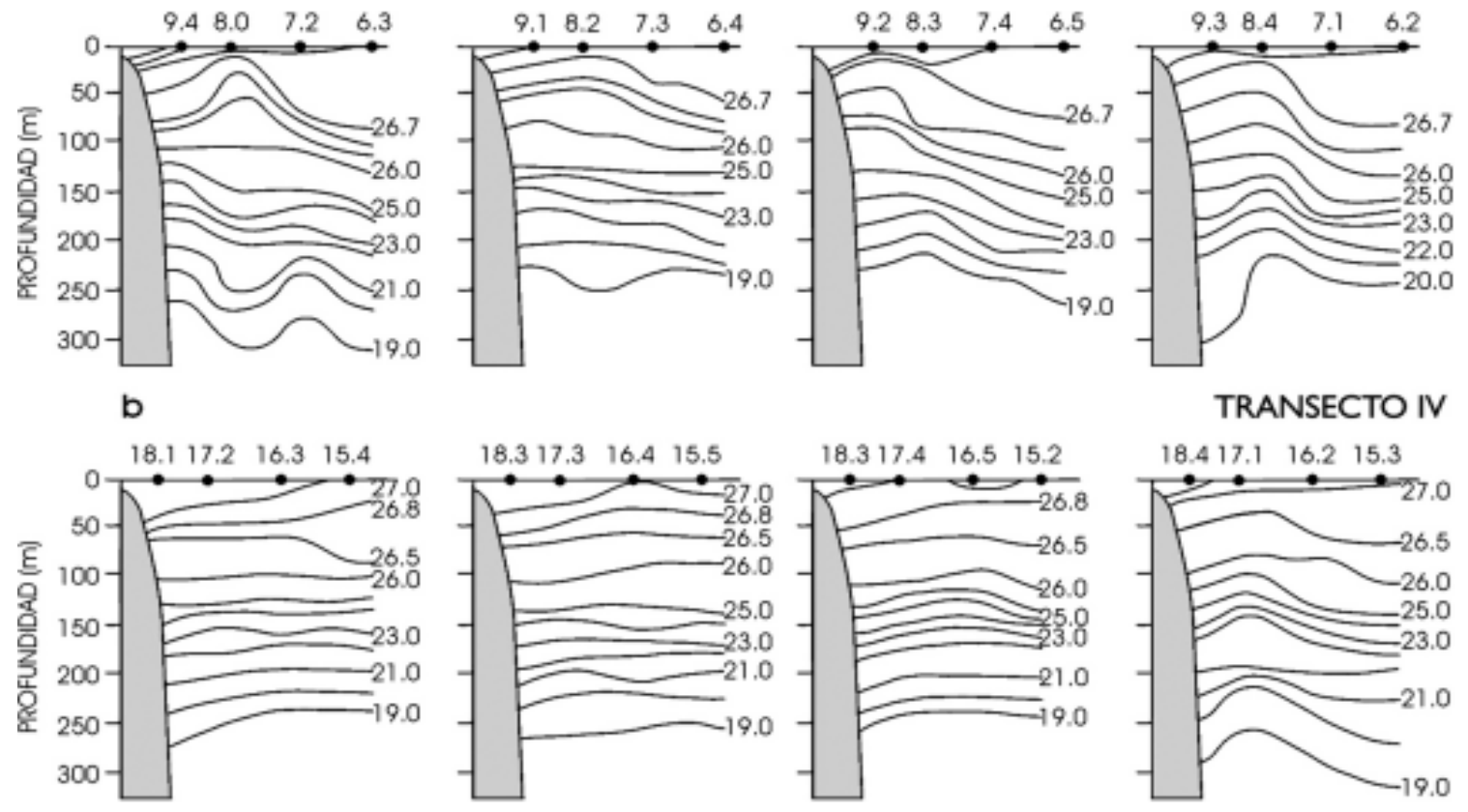

TRANSECTO IV

c
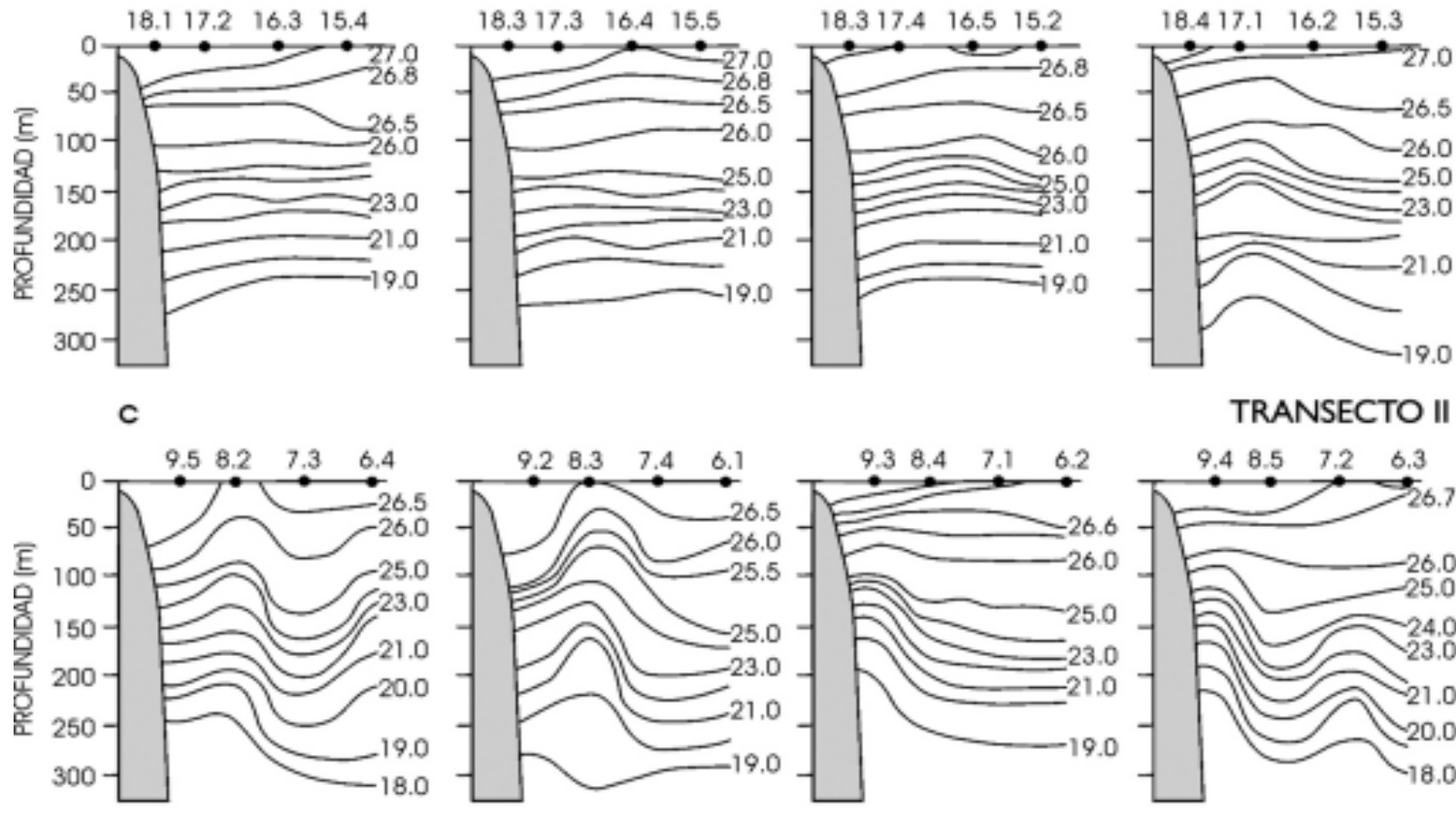

TRANSECTO ॥

d

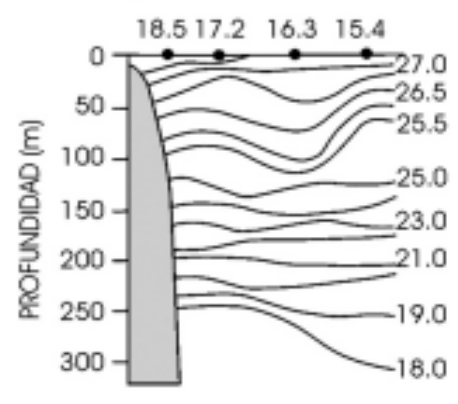

03:00 $\begin{array}{llll}18.2 & 17.3 & 16.4 & 15.5\end{array}$

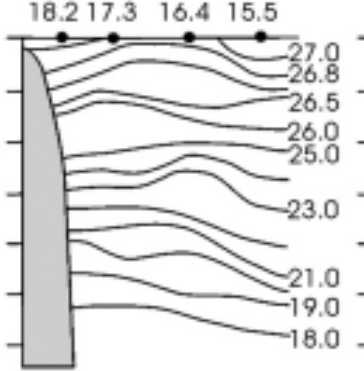

09:00

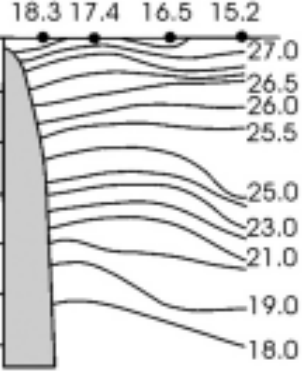

15:00

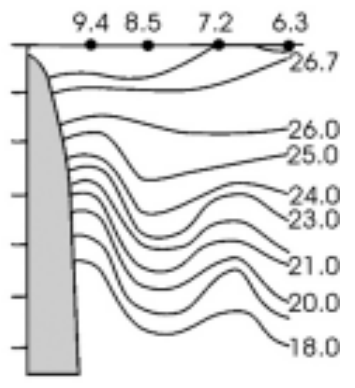

TRANSECTO IV

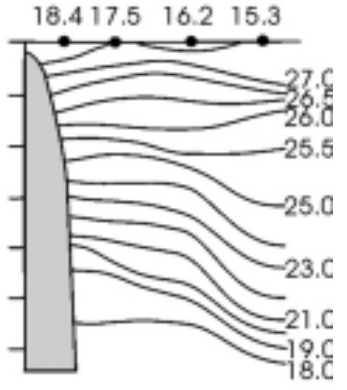

$21: 00$ hrs.

Figura 9. Distribución vertical de la temperatura $\left({ }^{\circ} \mathrm{C}\right)$ por hora de muestreo: 03:00, 09:00, 15:00 y 21:00 hora local (referidas al huso horario del meridiano 75,0 W). a) Transecto II en marzo 1994, b) Transecto IV en marzo 1994, c) Transecto II en abril 1996, d) Transecto IV en abril 1996.

Figure 9. Temperature vertical distribution $\left({ }^{\circ} \mathrm{C}\right)$ for 03:00, 09:00, 15:00 and 21:00 local time (referred to the use of the meridian 75.0 W). a) Transect II on March 1994, b) Transect IV on March 1994, c) Transect II on April 1996, d) Transect IV on April 1996. 
(Fig. 9c), las isotermas por encima de $100 \mathrm{~m}$ fueron más estables que en 1994, ya que el viento sopló por debajo de los $5 \mathrm{~m} \cdot \mathrm{s}^{-1}$ como promedio. En horas de la noche $(21 \mathrm{~h})$, al incrementarse el viento se inició nuevamente el ciclo que se observó desde finales de la tarde sobre la estación 8 (Figs. 9a y 9c).

En el transecto IV parece existir el mismo mecanismo aunque menos intenso. En horas de la madrugada la mezcla fue más marcada en la estación 17 en 1996. En la mañana y en la tarde, las isotermas tienden a profundizarse a medida que se acercan al talud, mientras que durante la noche se incrementa ligeramente la ondulación de las mismas, principalmente en la estación 17. Por lo tanto, el transecto II es más sinuoso y más claro el proceso de mezcla cercano al talud, mientras que en transecto IV no es muy acentuado.

La temperatura varió desde $27,6^{\circ} \mathrm{C}$ en superficie hasta $18^{\circ} \mathrm{C}$ en $250 \mathrm{~m}$ de profundidad. La isoterma de $25^{\circ} \mathrm{C}$ se ubicó como promedio entre $100-125 \mathrm{~m}$ en ambos cruceros y se evidenció una vez más, que las isotermas tienden a profundizarse a medida que se acercan a la plataforma, reafirmando el carácter de convergencia en las aguas cercanas al talud en esta zona.

\section{Estabilidad vertical}

Las figuras 10a y 10a' mostraron un gradiente de estabilidad menos fuerte, que osciló por encima de los $40 \mathrm{~m}$ y otro más fuerte que aumentó cerca de los $100 \mathrm{~m}$ al parecer, debido al aumento del gradiente de temperatura y salinidad provocado por el incremento de viento en la noche. En la mañana cuando disminuyó el viento (Figs. 10b y 10b'), la termoclina se hundió y disminuyó la estabilidad, esta vez en la capa cercana a $130 \mathrm{~m}$.

Las figuras 10c y 10c' muestran en la tarde que aumentó la velocidad del viento y con ello el transporte y el gradiente de la estabilidad cercana a la costa. Las figuras $10 \mathrm{~d}$ y $10 \mathrm{~d}$ ' indican la distribución vertical de la estabilidad durante la noche, cuando la velocidad del viento alcanzó mayor fuerza incidiendo en un aumento de la estabilidad.

La figura 10 (a, b, c, d,) indica que el primer gradiente de la estación 9 disminuyó hacia los 3040 m, mientras que en la estación 18 (Figs. 10a', b', c', d') a medida que avanzó el día el gradiente se hundió más, estando en la noche-madrugada muy cercana a superficie. Esta situación al parecer, explica por qué las máximas densidades se encontraron, generalmente, profundas y cercanas al talud durante el día y más cercanas a la superficie en la noche. En esta zona se produce una salida por el fondo de agua más densa con material particulado y arena del golfo de Batabanó llamado también "efecto cascada" que puede ser otro factor en la formación del gradiente superficial en los primeros $40 \mathrm{~m}$ en la estación 9.

La figura 11 muestra la distribución vertical de la estabilidad y de la PIT en horario de muestreo fijo en las estaciones costeras 9 y 18 durante el crucero de abril de 1996. El primer gradiente de la estabilidad en la estación 9 durante todos los horarios desaparece, con excepción de la madrugada que aparece cerca de los $20 \mathrm{~m}$, mientras que en la estación 18 se incrementa hacia la superficie y disminuye en la capa 20-40 m.

Las figuras 11a y 11a' indican el aumento de la estabilidad a consecuencia del aumento del viento de aproximadamente $30 \mathrm{~km} \cdot \mathrm{h}^{-1}$. Al disminuir la velocidad del viento a $18 \mathrm{~km} \cdot \mathrm{h}^{-1}$ entre fines de la mañana y la tarde (Figs. 11c y 11c'), la inercia del mar motivó que aumentara la mezcla sobre el talud, propiciando una disminución del gradiente de estabilidad a $80 \mathrm{~m}$ de profundidad y aumento del mismo hasta 6,5 unidades convencionales (u.c.) de densidad, la más alta registrada en el crucero.

Las figuras $11 \mathrm{~d}$ y $11 \mathrm{~d}$ ' muestran el horario nocturno donde aumentó ligeramente el viento y la estabilidad osciló en los 80 m y disminuyó hasta casi 4 (u.c.). La estación 18 no se presentó con las características de viento muy fuerte como en la estación 9, y su estabilidad fue similar a la del crucero de 1994.

\section{DISCUSIÓN}

La estabilidad vertical de la columna de agua es reconocida como una característica cuantitativa de las condiciones de equilibrio vertical del mar. Esta depende de la densidad que es función de la temperatura, salinidad y presión (Husby \& Nelson, 1982), siendo un elemento importante a la hora de estimar la disposición y límite de las capas de discontinuidad de la densidad en las zonas de convergencia y divergencia (Egorov, 1983). Además, en ocasiones sus características han sido relacionadas con el ciclo de vida de especies marinas, contribuyendo en la profundización de importantes mecanismos marinos (Husby \& Nelson, 1982; Shelton \& Hutchings, 1990; Bakun \& Parrish, 1991).

Lasker (1975) elaboró una hipótesis sobre la 


\section{Temperatura $\left({ }^{\circ} \mathrm{C}\right)$}
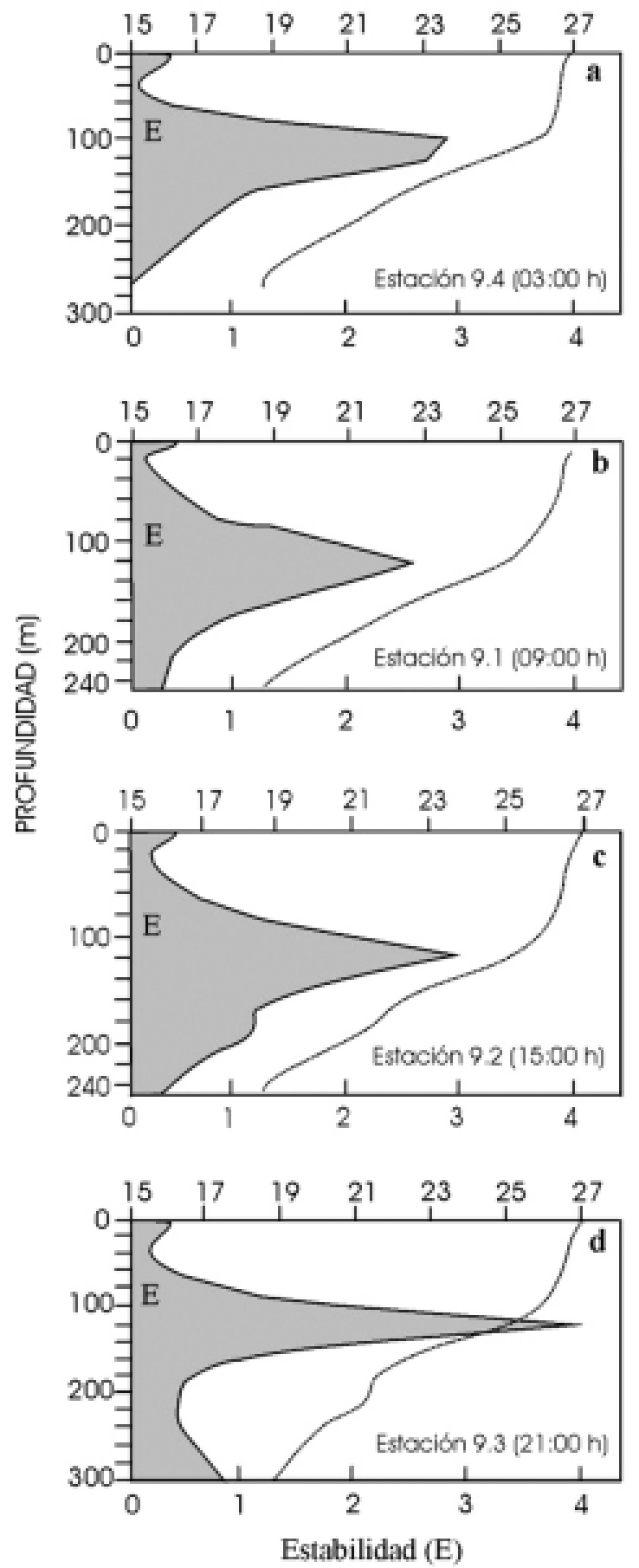

Temperatura (" $\mathrm{C})$
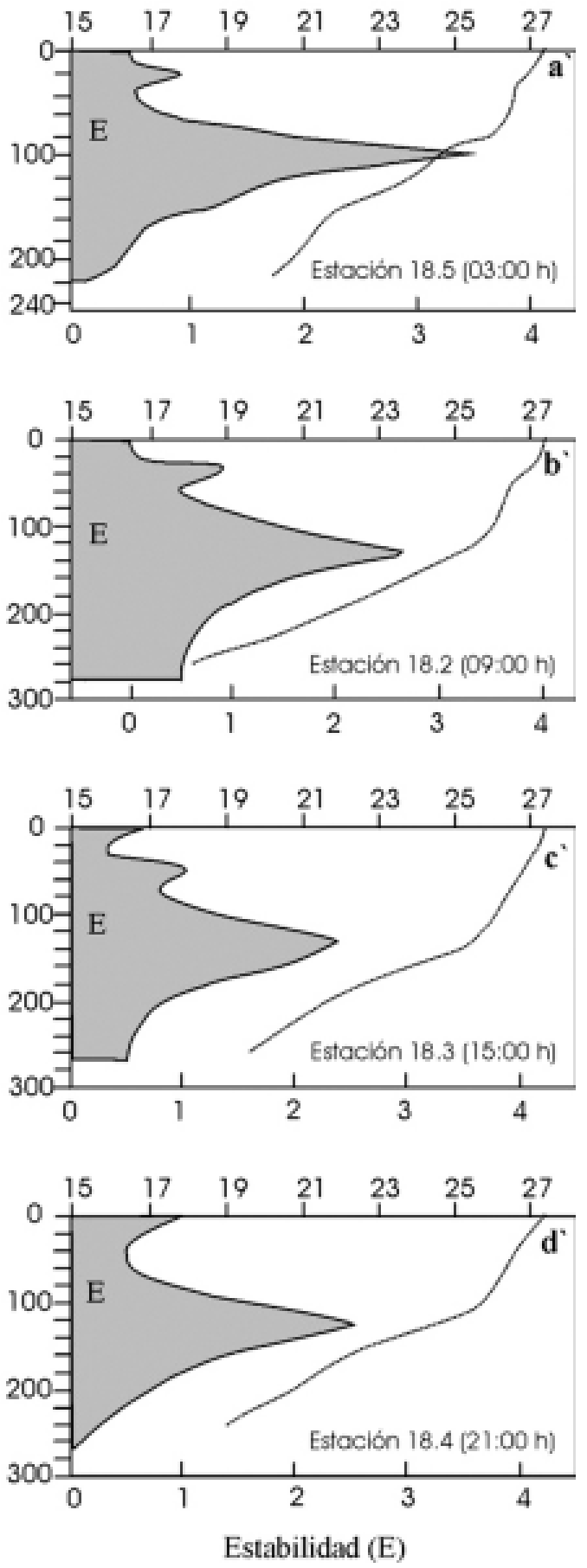

Figura 10. Perfil vertical de la estabilidad $(\mathrm{E})$ y temperatura $\left({ }^{\circ} \mathrm{C}\right)$ por hora de muestreo entre 0 y $300 \mathrm{~m}$ de profundidad. a, b, c y d representan la estación 9 (costera de 24 h), mientras que a', b', c' y d' pertenecen a los mismos horarios en la estación 18 (costera de 24 h), durante el crucero de marzo 1994.

Figure 10. Stability vertical profile $(\mathrm{E})$ and temperature $\left({ }^{\circ} \mathrm{C}\right)$ for sampling hours between 0 and $300 \mathrm{~m}$ of depth. a, b, c and d represent the station 9 (nearshore of $24 \mathrm{~h}$ ), while a', b', c' and d'belong to the same schedules in the station 18 (nearshore of $24 \mathrm{~h}$ ), during March 1994 cruise. 

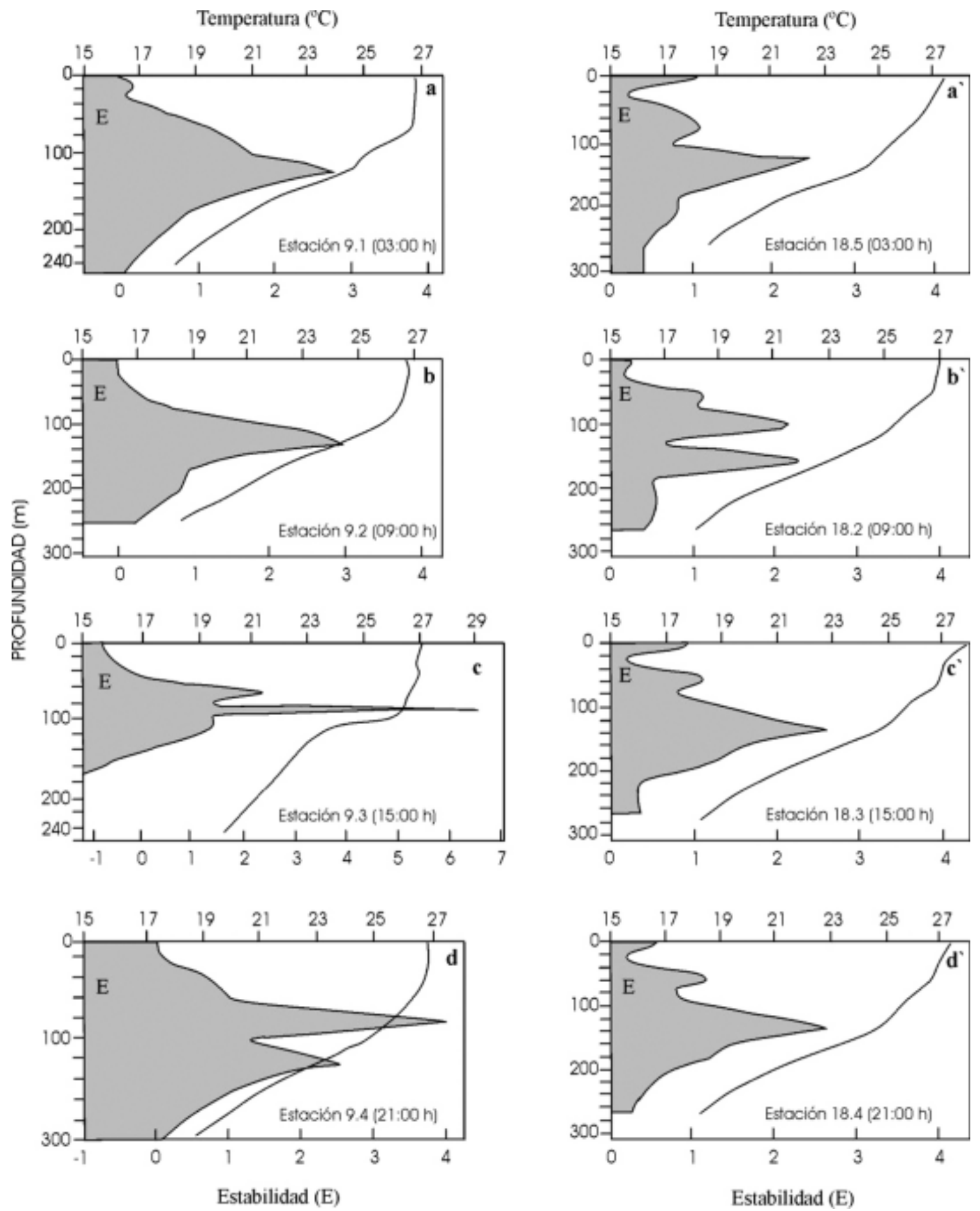

Estabilidad (E)

Figura 11. Perfil vertical de la estabilidad $(\mathrm{E})$ y temperatura $\left({ }^{\circ} \mathrm{C}\right)$ por hora de muestreo entre 0 y $300 \mathrm{~m}$ de profundidad. a, b, c y d representan la estación 9 (costera de 24 h), mientras que a', b', c' y d' pertenecen a los mismos horarios en la estación 18 (costera de 24 h), durante el crucero de abril 1996.

Figure 11. Stability vertical profile (E) and temperature $\left({ }^{\circ} \mathrm{C}\right)$ for sampling hours between 0 and $300 \mathrm{~m}$ of depth. a, b, c and d represent the station 9 (nearshore of 24 h), while a', b', c' and d' belong to the same schedules in the station 18 (nearshore of 24 h), during April 1996 cruise. 
sobrevivencia de larvas de anchoveta y concluyó que ésta fue dependiente de la estabilidad de la columna de agua. Blaxter \& Hunter (1982) demostraron en condiciones de laboratorio que la primera comida de la anchoveta requiere de altas concentraciones de plancton. Las concentraciones de larvas se encuentran en la parte superior de la columna de agua o zona eufótica, particularmente en los períodos de poco viento y reducida turbulencia (Lasker, 1975, 1978; Owen, 1989).

Peterman \& Brandford (1987) realizaron un análisis estadístico de los datos de viento y mortalidad larval, con datos de campo que confirmaron la hipótesis de Lasker. Existe una relación estadística significativa entre la tasa de mortalidad larval y la frecuencia de calmas, período de baja velocidad del viento que permite el mantenimiento y concentración de parches de alimentos. Sin embargo, las condiciones de calma completa no son ideales porque la columna de agua estratificada reduce el flujo vertical de nitratos dentro de la zona eufótica, reduciendo la posibilidad de un incremento de la nueva producción y de fitoplancton (Lewis et al., 1986; Hernández, 2001).

Las poblaciones de anchoveta del norte, centro y sur de California desovan principalmente en invierno y primavera (Smith 1972; Smith \& Richarson, 1977), en verano el desove es mínimo y se registran las mayores calmas de viento, y la columna de agua está más estratificada por el calentamiento (Husby \& Nelson, 1982).

En la plataforma suroccidental de Cuba, Alfonso et al. (1995b) encontraron que la distribución estacional del estadio I de la langosta $P$. argus, mostró una buena concordancia con la curva aproximada de reproducción y confirmó la mayor intensidad de reproducción en primavera-verano. Además, determinaron que el patrón principal de distribución de los filosomas muestran una disminución de larvas hacia fuera de la costa, patrón similar a lo encontrado para $P$. interruptus en California (Johnson, 1960; Campbell, 1986).

Las distribuciones verticales y horizontales registradas en el presente trabajo, junto a otros aspectos conocidos de la oceanografía regional, han sido consideradas por varios autores como las variables que pueden dar respuesta a los problemas de la actividad reproductiva de diferentes especies en una determinada región (Mendo et al., 1987; Mendelssohn \& Cury, 1987; Gargett, 1997; Gargett \& Marra, 2002).
La región de estudio, caracterizada por un constante hundimiento de las aguas debido a la persistencia de los alisios (Hernández et al., 1995), ofrece particularidades especiales de permanencia prolongada de larvas. Por una parte, la fuerte convergencia de las aguas hacia el talud concentran sobre esa franja, gran cantidad de partículas utilizadas en beneficio de las propias larvas, y por otra, el efecto físico de estas aguas que al llegar al talud arrastran en su hundimiento, nutrientes provenientes de ríos y pantanos que desembocan en el golfo de Batabanó. Este hundimiento produce un efecto de mezcla o falso afloramiento que mantienen los nutrientes resuspendidos por un período que varía según la intensidad del viento y las corrientes. En estas condiciones, las filosomas y/o larvas se sitúan en su máximo confort (Cayré, 1990), con temperaturas de alrededor de $\operatorname{los} 25^{\circ} \mathrm{C}$.

Las observaciones realizadas durante estos cruceros, reflejan la dinámica de la zona y la estrecha relación existente entre los procesos atmosféricos y las capas superficiales del océano. La fuerza del viento, su dirección y persistencia, la cobertura nubosa, la radiación solar y otros importantes aspectos meteorológicos, unido a las variaciones termohalinas del mar y corrientes imperantes en la zona, marcan concatenadamente fuertes gradientes de intercambio entre la plataforma y el talud que es aprovechado por la comunidad planctónica en general, para su supervivencia. Los resultados de las condiciones hidrometeorológicas registradas en los cruceros de 1994 y 1996, parecen estar más próximas a las ventanas óptimas ambientales (Cury \& Roy 1989; Gargett, 1997; Gargett \& Marra, 2002), en la sobrevivencia de larvas y posterior éxito del reclutamiento de la langosta espinosa $P$. argus, dos años después.

\section{CONCLUSIONES}

Las isotermas, profundidad de inicio de la termoclina, índice de transporte, turbulencia y estabilidad vertical de la columna de agua, obtenidos en ambos cruceros demuestran la veracidad de la hipótesis del mecanismo de acoplamiento entre el océano y la plataforma señalado por Hernández et al. (1995), así como la importancia de considerar la dirección y velocidad del viento como variable de fácil obtención en estudios hidrometeorológicos y pesqueros en una determinada región. Además, se observó, a través de las isotermas de TSM y de es- 
tabilidad vertical, que el proceso de mezcla que se origina en las estaciones fijas cercanas al talud, es responsable de la retención larvaria en esta región. Aunque los resultados de este trabajo se realizaron en la primavera de 1994 y 1996 donde el viento sopla con mayor rapidez, el mecanismo de hundimiento de las aguas en la región suroccidental de Cuba debe mantener un comportamiento parecido a lo largo del año, condicionado por la dirección y fuerza del viento imperante en la zona.

\section{AGRADECIMIENTOS}

El primer autor quiere dejar constancia de su agradecimiento al Dr. Julio A. Baisre, del Ministerio de la Industria Pesquera de Cuba por la confianza en la jefatura de los cruceros a bordo del BIC "Ulises". Los autores agradecen a la tripulación del BIC "Ulises", que sin su ayuda no se hubiera podido realizar el trabajo a bordo. A nuestros colegas del Instituto de Meteorología y del Centro de Investigaciones Pesqueras de Cuba. A los revisores del trabajo y en especial, a los Drs. Eleuterio Yáñez y Sergio Palma de la Pontificia Universidad Católica de Valparaíso por su entusiasmo en la publicación de este artículo.

\section{REFERENCIAS}

Alfonso, I., M.P. Frías, J. Baisre \& B. Hernández. 1995a. Distribución vertical de filosomas de langosta (Panulirus argus) y su relación con algunos factores hidroclimáticos al sur del golfo de Batabanó, Cuba. Rev. Cub. Inv. Pesq., 19(1): 3-9.

Alfonso I., M.P. Frías, J. Baisre \& A. Campos. 1995b. Distribución y abundancia de larvas de la langosta (Panulirus argus) en aguas alrededor de Cuba. Rev. Cub. Inv. Pesq., 19(1): 10-15.

Bakun, A. 1973. Coastal upwelling indices, west coast of North America, 1946-71. US. Dep. Comm. NOAA. Tech. Rep. NMFS-SSRF 671: 103 pp.

Bakun, A. \& R.H. Parrish. 1991. Comparative studies of coastal pelagic fish reproductive habitats: the anchovy (Engraulis anchoita) of the Southwestern Atlantic. ICES. J. Mar. Sci., 48: 343-361.

Bakun, A., V. Christensen, C. Curtis, P. Cury, M.H. Durand, D. Husby, R. Mendelssohn, J. Mendo, R. Parrish, D. Pauly \& C. Roy. 1992. The climate and eastern ocean system project. NAGA ICLARM Q., 15(4): 26-30.

Banner, M. 1990. On the influence of the sea state on the winds stress. Int. TOGA. Sc. Cont. Proc. WCRP., 43: 139-144.

Blaxter, J.H.S. \& J.R. Hunter. 1982. The biology of clupeid fishes. En: J.H.S. Blaxter, F.S. Rusell \& M. Young (eds.). Adv. Mar. Biol., 20: 1-223.

Bogdanov, D.V. 1966. Algunos resultados de las investigaciones oceanográficas Prim. Conf. Sob. Inv. Pesq. Cub. Sov. (1964-66) INP/CIP, La Habana. pp. 219-237.

Cayré, R. 1990. Les migrations: un comportement déclenché et guide par l'environnant. ICCAT. 32(1): 158-163.

Campbell, A. 1986. Migratory movement ovigerous lobster, (Homarus americanus), tagged of Gran Manan, eastern Canada. Can. J. Fish. Aquat. Sci., 43(11): 2197-2205.

Campos, A. 1989. Radiación solar. Clima. Nuevo Atlas Nacional de Cuba. Academia de Ciencias de Cuba, Instituto Cubano de Geodesia y Cartografía. Ediciones del Instituto Geográfico Nacional de España, Madrid, pp. VI.1-3.

Charnock, H. 1955. Wind stress on a water surface. Quart. J. Roy. Met. Soc., 81: 639-640.

Charnock, H. 1981. Air-sea interaction. En: B.A. Warren \& C. Wunsch (eds.). Evolution of physical oceanography. MIT Press, Boston, Mass. pp. 482503.

Cury, P. \& C. Roy. 1989. Optimal environmental window and pelagic fish recruitment success in upwelling areas. Can. J. Fish. Aquat. Sci., 46(4): 670-680.

Egorov, N.I. 1983. Oceanografía física. Vneshtorgizdat, Moscú, 553 pp.

Ekman, V.W. 1905. On the influence of earth's rotation on ocean current. Ark. f. Mat. Astron. Och. Fysik., 12(11): 1-53.

Elizarov, A. \& J.A. Gómez. 1966. Sobre la dinámica de las aguas que rodean a Cuba. INP/CIP, Cuba, $11 \mathrm{pp}$.

Emilsson, I. \& J.A. Gómez. 1971. La red de estaciones fijas alrededor de Cuba y notas sobre el resultado de los tres primeros cruceros. UNESCO, Paris. 
Coloquio Investigaciones de Recursos Marinos del Caribe y Regiones Adyacentes, pp. 61-64.

Fofonoff, N.P. \& R.B. Montgomery. 1955. The equatorial undercurrent in the light of vorticy equation. Tellus, 17(4): 518-521.

Francis, R.C. \& S.R. Hare. 1994. Decadal-scale regime shifts in the large marine ecosystems of the North-east Pacific: a case for historical science. Fish. Oceanogr., 3: 279-291.

Gargett, A.E. 1997. Physics to fish: interactions between physics and biology on a variety of scales. Oceanography, 10(3): 128-131.

Gargett, A.E. \& J. Marra. 2002. Effects of upper ocean physical processes (turbulence, advection and air-sea interaction) on oceanic primary production. pp. 19-49 En: A.R. Robinson, J.J. McCarthy \& B.J. Rothschild (eds.). The sea, ideas and observations on progress in the study of the seas. John Wiley \& Sons, London, Vol. 12, 634 pp.

Gómez, J.A. 1979. Corrientes geostróficas alrededor de Cuba. Rev. Cub. Inv. Pesq., 4(3): 89-102.

Hernández, B., R. Puga, R. Piñeiro, A. Peñate, I. Alfonso \& J. Baisre. 1995. Transport, turbulence and hypothetical ocean-shelf coupling mechanism in Punta del Este, southwestern Cuba. Rev. Cub. Inv. Pesq., 19(1): 67-72.

Hernández, B. 2001. Variabilidad interanual de la producción nueva en la Corriente de California. Tesis de Magíster en Ciencias en Ecología Marina. CICESE, Ensenada, 100 pp.

Hernández, B. 2002. El Niño-Oscilación del Sur (ENOS) y los frentes fríos que arriban a la región occidental cubana. Invest. Mar., Valparaiso, 30(2): 3-19.

Husby, D.M. \& C.S. Nelson. 1982. Turbulence and vertical stability in the California Current. CalCOFI Rep., 23: 113-129.

Instituto de Oceanología (IDO). 1989. Paquete oceanográfico DATAOCEAN. Instituto de Oceanología, Academia de Ciencias de Cuba.

Johnson, M.W. 1960. Production and distribution of larvae of spiny lobster (Panulirus interruptus) (Rondall) with records of (P. gracilis) Streets. Bull. Scripps Inst. Oceanogr., 7(6): 413-462.

Kucas, S.T., Jr. 1986. Species profiles: life histories and environmental requirements of coastal fishes and invertebrates (Pacific Southwest) northern anchovy. U.S. Fish. Wildl. Serv. Biol. Rep. 82(11.50). U.S. Army Corps of Engineers, TR EL82-4, $11 \mathrm{pp}$.

Lasker, R. 1975. Field criteria for survival of anchovy larvae: The relationship between inshore chlorophyll maximum layers and successful first feeding. U.S. Fish. Bull., 73: 453-462.

Lasker, R. 1978. The relation between oceanographic conditions and larval anchovy food in the California current: identification of factors contributing to recruitment failure. Rapp. P.-V. Réun. Cons. int. Explor. Mer, 173: 212-230.

Lewis, M.R., W.G. Harrison, N.S. Dakey, D. Hebert \& T. Platt. 1986. Vertical nitrate fluxes in the oligotrophic ocean. Science, 234: 870-873.

Mendelssohn, R. \& P. Cury. 1987. Fluctuation of a fortnightly abundance index of the Ivoirian coastal pelagic species and associated environmental conditions. Can. J. Fish. Aquat. Sci., 44: 408-421.

Mendo, J., L. Pizarro \& S. Castillo. 1987. Monthly turbulence and Ekman transport indexes 1953 to 1985, base don local wind records from Trujillo and Callao, Peru, pp.75-88. En. D. Pauly \& I. Tsukayama (eds.). The Peruvian anchoveta and it's upwelling ecosystem: three decades of change. Instituto del Mar del Peru (IMARPE), Callao, Peru; Deustsche Gesellschaft fur Technische Zusammenarbeit (GTZ), GmbH, Eschbom, Federal Republic of Germany; and International Center for Living Aquatic Resources Management (ICLARM), Manila, Philippines. ICLARM. Stud. Rev., 15: 1-351.

Mysak, L.A. 1986. El Niño, interannual variability and fisheries in the northeast Pacific Ocean. Can. J. Fish. Aquat. Sci., 43: 464-497.

Munk, W.H. \& E.R. Anderson. 1948. Notes on a theory of the termocline. J. Mar. Res., 7(3): 276295.

Owen, R.W. 1989. Microscale and fine scale variations of small plankton in coastal and pelagic environmental. J. Mar. Res., 47: 197-240.

Pauly, D. 1989. An eponym for Reuben Lasker. U.S. Fish. Bull., 87(3): 383-384.

Pérez, A. \& A. Alfonso. 1991. Programa BAKUN. CIPSOFT. No.1 Copyright. La Habana. 
Peterman, R.M. \& M.J. Brandford. 1987. Wind speed and mortality rate of marine fish the northern anchovy (Engraulis mordax). Science, 235: 354356.

Rodríguez, M. \& M. Ballester. 1985. Cronología de los "sures" que han afectado a la mitad occidental de Cuba desde la temporada 1916-17 hasta la de 1983-84. Rep. Inv., 10 ACC: 15 pp.

Rodríguez, E., I. Victoria, A. Rodríguez del Rey \& L. Vila. 1990. Corrientes geostróficas alrededor de Cuba: Consideraciones Pesqueras. II Congreso de Ciencias del Mar. La Habana,19 pp.

Rossov, V.V. 1967. Sobre el sistema de corrientes del Mediterráneo Americano. Instituto de Oceanología. ACC, 2(1): 31-49.

Seguí, M.E. 1989. Frecuencia de días despejados. Clima. Nuevo Atlas Nacional de Cuba. Academia de Ciencias de Cuba. Instituto Cubano de Geodesia y Cartografía. Ediciones del Instituto Geográfico Nacional de España, Madrid, pp. VI.1-3.

Recibido: 4 enero 2001; Aceptado: 10 abril 2003
Shelton, P.A. \& L. Hutchings. 1990. Ocean stability and anchovy spawning in the Southern Benguela Current region. U.S. Fish. Bull., 88(2): 323-338.

Siam, C. \& B. Hernández. 1981. Características oceanográficas en dos polígonos al sur de Cuba. Rev. Cub. Inv. Pesq., 6(1): 71-96.

Smith, P.E. 1972. The increase in spawning biomass of northern anchovy (Engraulis mordax). U.S. Fish. Bull., 70: 849-874.

Smith, P.E. \& S.L. Richardson. 1977. Standard techniques for pelagic fish eggs and larval surveys. FAO Fish. Tech. Paper, 175: 1-100.

Tápanes, J.J. 1963. Afloramientos y corrientes cercanas a Cuba. Centro de Investigaciones Pesqueras, La Habana, CIP-Contribución, 17: 1-29.

Vega, R. 1989. Clima. Nuevo Atlas Nacional de Cuba. Academia de Ciencias de Cuba. Instituto Cubano de Geodesia y Cartografía. Ediciones del Instituto Geográfico Nacional de España, Madrid, pp. VI.1-3. 